Ann. Zootech., 1978, 27 (3), 409-42I.

\title{
Glucose, sirop de glucose \\ à haute teneur en fructose (S.G.H.T.F.), ou saccharose dans les aliments de sevrage précoce du porcelet : influence sur l'agglomération et l'appétibilité
}

\author{
A. AUMAITRE, J. P. MELCION $\left(^{*}\right)$, P. VAISSADE et B. SEVE \\ avec la collaboration technique de Jany Peiniau, A. Lapanouse et H. Varo \\ Station de Recherches sur l'Élevage des Porcs, \\ Centre national de vecherches zootechniques, I.N.R.A., \\ $7835^{\circ}$ Jouy-en-Josas (France) \\ (*) Laboratoire de Technologie des Aliments des Animaux, I.N.R.A., \\ Chemin de la Géraudière, 44072 Nantes Cedex (France)
}

\section{Résumé}

L'appétibilité conférée aux aliments des porcelets par l'incorporation de substances à pouvoir sucrant (saccharose, glucose, saccharine, sirop à haute teneur en fructose ou S.G.H.T.F.) a été mesurée au cours de 4 expériences utilisant 252 animaux sevrés à 35 jours et 80 animaux sevrés à $2 \mathrm{~L}$ jours.

L'aliment contenant du saccharose est toujours préféré par les animaux qui peuvent effectuer un choix; les aliments contenant du glucose sont délaissés quelle que soit la dose (5 ou io p. Ioo). Le remplacement de 5 p. ıoo de saccharose par une quantité équivalente de S.G.H.T.F. augmente le rendement au pressage de $\mathrm{r}_{4} \mathrm{p}$. Ioo. Cependant il fait baisser dangereusement la dureté des agglomérés lorsqu'on en incorpore Io p. IOO $\left(\mathrm{I}, 9 \mathrm{bar} / \mathrm{cm}^{-1}\right.$ contre $3,6 \mathrm{bar} / \mathrm{cm}^{-1}$ pour l'aliment témoin). Malgré sa composition en oses identique, il n'attire pas le porcelet qui préfère les aliments à base de saccharose. Le S.G.H.T.F. ne semble donc présenter qu'un intérêt limité dans 1'aliment des porcelets.

Le choix des animaux s'opère précocement, en général pendant l'allaitement, et la préférence s'installe très rapidement et définitivement avant ou après sevrage. Toutefois, les observations effectuées sur des lots d'animaux comparables ne disposant que d'un seul aliment montrent que les quantités consommées sont identiques lorsqu'elles contiennent du saccharose ou du glucose. De tels résultats suggèrent que la notion d'appétibilité déterminée par la présentation simultanée de deux aliments reste un peu un artifice expérimental. Leur extrapolation à la recommandation d'incorporer des produits à pouvoir sucrant (saccharose notamment) aux aliments de sevrage précoce nous paraît ainsi discutable.

En effet, les sensations buccales que provoque son ingestion ne permettent pas de modifier, donc de faire augmenter le montant de la prise alimentaire chez le porcelet sevré précocement.

\section{I. - Introduction}

L'appétibilité d'un aliment selon LE MAGNEN (I962) désigne " le fait qu'il est plus ou moins consommé en réponse à un état physiologique de l'animal ou appétence pour ce produit, déterminée par l'activité sensorielle buccale de l'aliment au cours de son ingestion $"$. 
Chez le porcelet allaité qui malgré une insuffisance des apports nutritionnels maternels, répugne à consommer précocement l'aliment sec offert à volonté, cette notion a été souvent retenue. C'est ainsi que l'on a cherché et que l'on cherche encore à rendre les aliments du porcelet les plus appétibles possible afin qu'il s'affranchisse plus vite de la dépendance maternelle et se prépare à un sevrage précoce.

Pendant longtemps, en raison d'une production mondiale élevée, on a démon . tré expérimentalement que le saccharose incorporé à 1'aliment entre 5 et $40 \mathrm{p}$. Ioo conférait à l'aliment une appétibilité élevée par rapport aux aliments non sucrés (Lewis, Catron et Combs, i953; Nelson, Hazel et Catron, i953; SalmonLEGAGNEUR et FÉVRIER, I956; COMBS et WALLACE, I959). La recherche d'autres produits édulcorants lorsque le sucre est devenu rare et cher a été envisagée, en essayant sans grand succès la mélasse ou la saccharine (NotzoLd et al., I955). Ce dernier résultat est imputable au faible attrait exercé chez le Porc par la saccharine incorporée à l'eau de boisson, au contraire du saccharose ou du glucose (KENNEDY et BALDWIN, I972).

Le saccharose entraîne toujours une augmentation de l'appétibilité de l'aliment qui en contient, lorsque les auteurs offrent un choix au porcelet; mais l'application de ce résultat dans la pratique de l'alimentation est délicate. Aussi avonsnous voulu comparer par ailleurs les quantités consommées suivant la nature du produit édulcorant et son taux d'incorporation, entre groupes de porcelets recevant un seul aliment.

Enfin, un nouveau produit "sucrant " est apparu récemment sur le marché, issu de l'isomérisation partielle en fructose d'un sirop de glucose et appelé "High Fructose Corn Syrup " ou Sirop de Glucose à Haute Teneur en Fructose (S.G.H.T.F.), il peut être disponible en grande quantité et à faible prix (IIsuka et al., r972). Nous avons voulu vérifier l'influence de l'incorporation d'un tel produit dans les aliments. En effet, outre son intérêt zootechnique, sa composition en oses très voisine de celle du saccharose peut permettre d'entrevoir l'origine physiologique du comportement alimentaire du porcelet. On a recherché dans un premier temps. les effets propres des stimulations gustatives de ce nouveau produit sur la prise préférentielle d'aliments qui en contiennent, en comparaison avec le saccharose.

\section{II. - Matériel et méthodes}

\section{I. - Animaux}

332 porcelets sevrés, soit à 35 jours $(252)$, soit à 2 I jours (80) ont été affectés dans 4 expériences successives (tabl. I). Des animaux sevrés à 35 jours sont répartis en un seul lot (expérience $\mathrm{I}$ ) ou en 3 groupes d'animaux contemporains constitués à partir de deux portées (expérience 2 ). Des portées entières de porcelets sous la mère, puis sevrés à 5 ou 3 semaines sont utilisées pour la mesure de l'appétibilité des aliments contenant le sirop de glucose pendant les deux périodes encadrant le sevrage (expériences 3 et 4 ).

\section{2. - Aliments}

Les aliments de sevrage à 35 jours (expériences $I, 2$ et 3 ) sont à base d'orge, de tourteau de soja, de farine de hareng et de poudre de lait écrémé en quantité constante. Seules varient les quantités ou la nature des glucides par rapport au 
TABLEAU I

Schéma des expériences

Experimental design used in the 4 successive experiments

\begin{tabular}{|c|c|c|c|}
\hline $\begin{array}{l}\text { Expérience } \\
\text { Experiment }\end{array}$ & $\begin{array}{l}\text { Animaux } \\
\text { Animals }\end{array}$ & $\begin{array}{l}\text { Régimes composés } \\
\text { Experimental diets }\end{array}$ & $\begin{array}{l}\text { Dispositif expérimental } \\
\text { Experimental design }\end{array}$ \\
\hline $\mathbf{I}$ & $\begin{array}{l}\text { Sevrés; } 4 \text { répétitions de } 6 \text { à } \\
8 \text { porcelets }(27) \\
\text { entre } 6 \text { et } 9 \text { semaines }\end{array}$ & $\begin{array}{l}5 \% \text { saccharose } \\
5 \% \text { glucose } \\
5 \% \text { amylacés + saccharine }\end{array}$ & $\begin{array}{l}3 \text { aliments offerts au choix } \\
\text { à tous les animaux }\end{array}$ \\
\hline 2 & $\begin{array}{l}\text { Sevrés; } 7 \text { répétitions de } 4 \\
\text { porcelets par lot }\left(8_{4}\right) \\
\text { entre } 6 \text { et } 9 \text { semaines }\end{array}$ & $\begin{array}{l}5 \% \text { saccharose } \\
5 \text { ou ro } \% \text { glucose }\end{array}$ & I seul aliment offert par lot \\
\hline $3(1)$ & $\begin{array}{l}\text { Allaités; puis sevrés } 14 \text { por- } \\
\text { tées de porcelets (14 I). } \\
\text { Sevrés à } 35 \text { jours } \\
\text { entre } 7 \text { et } 42 \text { jours }\end{array}$ & $\mid \begin{array}{l}5 \% \text { saccharose } \\
\left.7,3 \text { ou } \text { I } 2 \% \text { S.G.H.T.F. }{ }^{2}\right)\end{array}$ & 3 aliments offerts au choix \\
\hline $4(1)$ & $\begin{array}{l}\text { Allaités puis sevrés; } 9 \text { por- } \\
\text { tées de porcelets }(80) \\
\text { Sevrés à } 2 \text { I jours } \\
\text { entre } 7 \text { et } 35 \text { jours }\end{array}$ & $\begin{array}{l}\text { I4, IO, } 3 \text { ou } 7 \% \text { saccharose } \\
\text { o, } 5 \text { ou Io } \% \text { S.G.H.T.F. }\end{array}$ & \\
\hline
\end{tabular}

(1) Expériences 3 et 4 : observations sous la mère puis après sevrage, pendant une et deux semaines (Experiments 3 and 4 were performed with suckling piglets, and continued with weaned piglet).

(2) Sitop de Glucose à Haute Teneur en Fructose (High Fructose Corn Syrup H.F.C.S.).

TABLEAU 2

Composition des aliments de sevrage à 5 semaines, à base de saccharose ou de glucose (expériences $I$ et 2 )

Composition of the starters used for weaning at 5 weeks, based on sucrose or glucose (experiments $I$ and 2 )

\begin{tabular}{|c|c|c|c|c|c|c|}
\hline \multirow{2}{*}{$\begin{array}{l}\text { Composition centésimale } \\
\text { Composition of the diets }\end{array}$} & \multirow{2}{*}{\multicolumn{3}{|c|}{$\begin{array}{l}\text { Expérience } \mathrm{I} \\
\text { Experiment } I\end{array}$}} & \multicolumn{3}{|c|}{$\begin{array}{l}\text { Expérience } 2 \\
\text { Experiment } 2\end{array}$} \\
\hline & & & & Lot & Lot 3 & Lot 3 \\
\hline 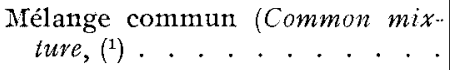 & 66 & 66 & 66 & 66 & 66 & 66 \\
\hline Manioc (Cassava) . . . . . . . & 29 & 29 & 29 & 29 & 29 & 24 \\
\hline $\begin{array}{l}\text { Saccharose (Sucrose)..... } \\
\text { Glucose (Glucose). } \\
\text { Amylacés }+ \text { saccharine (Starch } \\
\text { products }+ \text { saccharin) }\left({ }^{2}\right)\end{array}$ & -5 & $\begin{array}{r}- \\
5 \\
-\end{array}$ & - & $\frac{5}{-}$ & $\begin{array}{r}- \\
5 \\
-\end{array}$ & IO \\
\hline
\end{tabular}

(1) Pour Ioo kg d'aliment de sevrage (Per Ioo kg of starter) :

Orge : 27 , Suif : 4 , Tourteau de soja " 45 ": 20, Farine de poisson de Norvège : 6 , Poudre de lait écrémé : 5, Minéraux, vitamines, antibiotiques : 4 (Barley: 27, Tallow: 4, Soyabean oil meal " 45 ": 20 , Fish meal: 6, Dried skim-milk: 5, Mineral, vitamins, antibiotics : 4.

(2) Amylacés : Mélange amidon de maïs +- maltodextrine et saccharine pure apportant 1 ro ppm de saccharine ou I Io $\mathrm{mg} / \mathrm{kg}$ (Starch products containing maize starch, maltodextrin 0.22 p. Ioo of saccharin to provide IIO ppm of saccharin i.e. IIO $\mathrm{mg} / \mathrm{kg}$ of final starter). 
TABLEAU 3

Composition des aliments de sevrage $\grave{a} 3$ et à 5 semaines, contenant des proportions variables de sirop de glucose à haute teneur en fructose (S.G.H.T.F.) utilisées dans les expériences 3 et 4

Composition of starters for weaning at 3 or 5 weeks, containing high fructose corn syrup (H.F.C.S.) used in experiments 3 and 4

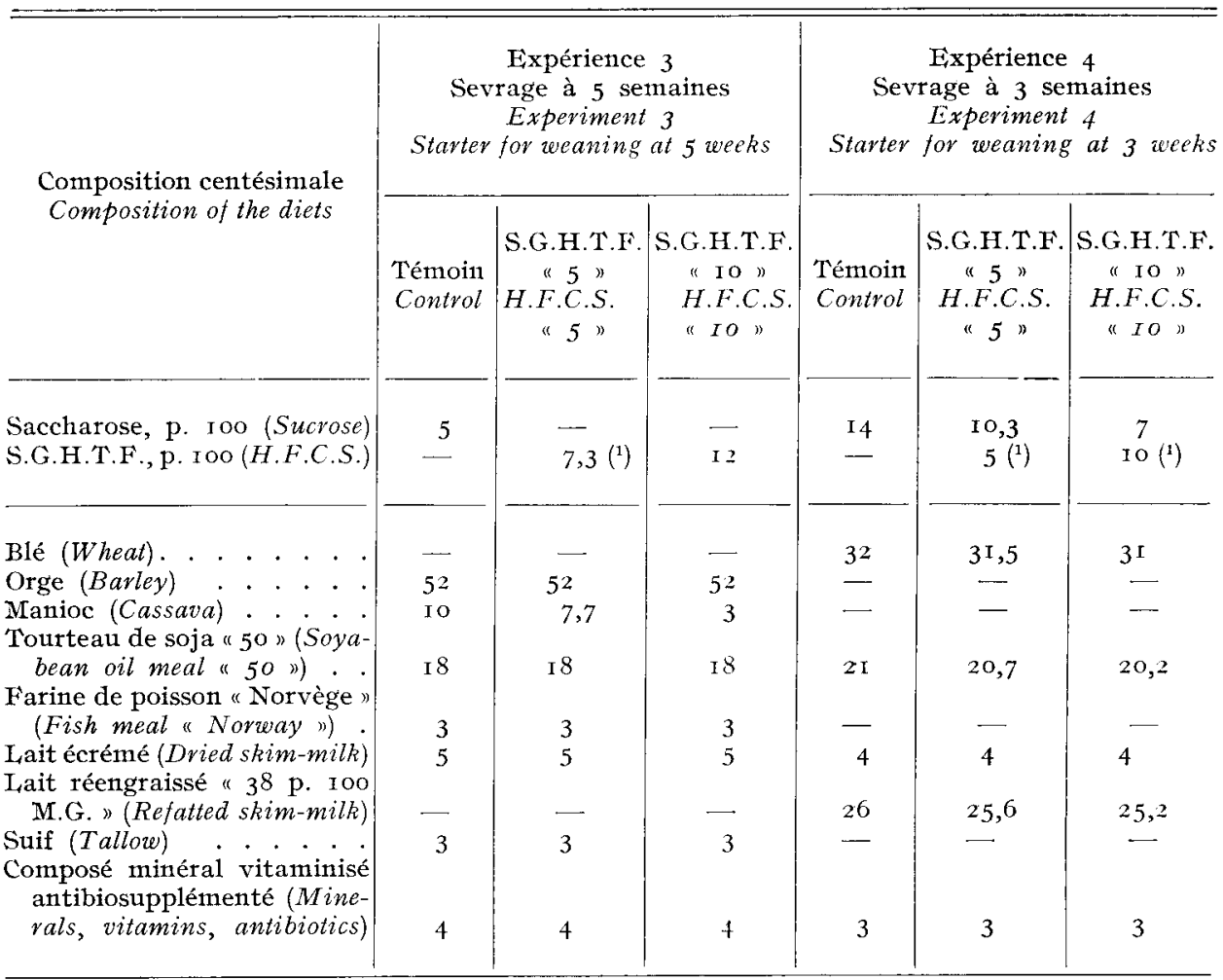

(1) Remplace une quantité équivalente de saccharose (In replacement by the same amount of sucrose).

manioc (tabl. 2 et 3 ). On mesure l'appétibilité comparée du glucose par rapport à celle du saccharose incorporé à 5 p. Ioo, que l'animal sait reconnaître parfaitement lorsqu'il a plusieurs aliments à sa disposition (SALMON-LEGAGNEUR et FÉvrIER, r955). I'effet d'appétibilité de la saccharine a été mesuré en considérant que cette demière a un pouvoir sucrant égal à 500 fois celui du saccharose (expérience I). On a ensuite distribué l'un des aliments précédents à chaque lot d'animaux sevrés (expérience 2). On a comparé l'appétibilité du sirop de glucose à haute teneur en fructose (S.G.H.T.F.) incorporé en quantité équivalente ou supérieure au saccharose (expérience 3). Le sirop de glucose a été introduit en remplacement de quantités croissantes de sucre pour mesurer l'appétibilité d'un aliment distribué avant et après le sevrage à $2 \mathrm{I}$ jours (expérience 4 ).

Dans toutes les expériences, les aliments sont agglomérés avec la même presse (GONDARD VT 400) en cylindres de $2,5 \mathrm{~mm}$ de diamètre. Des essais d'agglomé- 
ration ont été effectués séparément, à l'aide d'une presse CPM de laboratoire munie d'une filière de $2,4 \mathrm{~mm}$ de diamètre sur $38 \mathrm{~mm}$ d'épaisseur. Les conséquences technologiques de l'incorporation du sucre ou du sirop de glucose sont étudiées (expérience 3) par rapport à un aliment témoin à base de saccharose aggloméré soit à sec soit avec addition de 2 p. Ioo d'eau.

On a incorporé le S.G.H.T.F. sous forme liquide à l'aliment après avoir contrôlé sa composition. On a recherché sa présence dans les agglomérés après fabrication, rebroyage et extraction à l'eau. Un dosage qualitatif rapide a été tenté à l'aide de techniques colorimétriques automatisées permettant de doser les sucres réducteurs totaux réduisant le ferricyanure, le glucose vrai (glucose oxydase) ou le fructose vrai (résorcinol alcoolique) dans les conditions décrites par VAISSADE, AUMAITRE et RERAT (I973).

\section{3. - Expression des résultats}

Les principaux résultats concernent d'abord l'analyse chimique élémentaire. Après mélange, on a mesuré le rendement électrique et le débit de la presse, ainsi que certaines caractéristiques physiques des aliments agglomérés, friabilité (PFosT

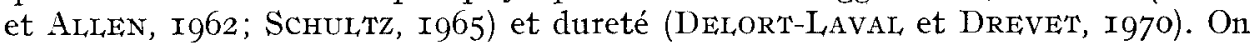
exprime l'appétibilité des aliments par le rapport des quantités hebdomadaires d'aliment expérimental consommées, à celles de l'aliment témoin, lorsque les animaux peuvent porter leur choix sur plusieurs aliments. Les performances de croissance sont exprimées en moyenne pour l'ensemble de la période expérimentale et par lot.

\section{III. - Résultats}

\section{I. - Caractérisation et détection de la présence de sirop de glucose (S.G.H.T.F.) dans les aliments complexes}

Le sirop de glucose isomérisé utilisé contenait une proportion sensiblement égale de glucose et de fructose. Toutefois, une fraction de la matière sèche essentiellement glucidique n'est pas détectée (tab1. 4). En effet, pas plus la quantité de sucres réduisant le ferricyanure de potassium, que la somme glucose + fructose ne rendent compte de la totalité de la matière organique du sirop qui ne contient qu'une très faible proportion de minéraux (0,03 p. Ioo). Dans les aliments de sevrage, le dosage des sucres réducteurs ainsi que celui du glucose rendent compte de l'augmentation $\mathrm{du}$ taux d'incorporation du sirop, contrairement au dosage du fructose.

\section{2. - Conséquences technologiques de l'incorporation de S.G.H.T.F.}

Lorsqu'on étudie 1'agglomération à ampérage constant (4,0-4,2 A), l'énergie spécifique consommée varie fortement: elle diminue de $27 \mathrm{p}$. Ioo avec ro p. Ioo de S.G.H.T.F. par rapport au témoin pressé à sec (tabl. 5). Le débit de la presse est accru de $55 \mathrm{p}$. I oo et l'échauffement du produit est moindre. La température à laquelle sont portés les agglomérés est abaissée de $8^{\circ} \mathrm{C}$. La dureté des agglomérés est surtout diminuée par une addition de Io p. Ioo de S.G.H.T.F. (tab1. 5) qui 


\section{TABIEAU 4}

\section{Composition du sirop de glucose isomérisé et des aliments préparés (expérience 3) pour les porcelets (en p. Ioo du produit frais) \\ Proximate chemical analysis of H.F.C.S. (High Fructose Corn Syrup) and starters (in experiment 3) (as p. roo of raw product)}

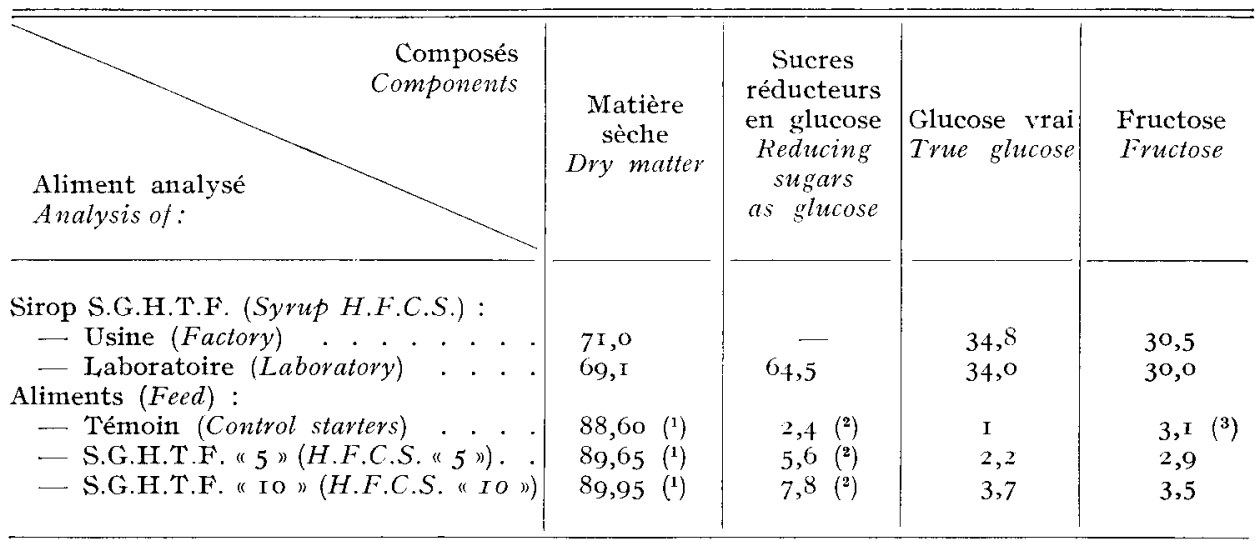

(1) Au cours de l'utilisation par les animaux (During feeding of the animals).

(2) Réponse globale (glucose + fructose libres + lactose).

(3) Réponse conjointe du fructose libre et du saccharose à la réaction (VAISsade ct al., I973).

apporte environ 3 p. roo d'eau. La friabilité de l'aliment est excessive pour l'aliment contenant un fort pourcentage ( 4 p. IOo) de saccharose lorsqu'il est aggloméré à sec, alors qu'elle reste très faible dans les autres cas.

\section{3. - Appétibilité des principaux aliments contenant du sucre ou du glucose}

L'aliment renfermant $5 \mathrm{p}$. Ioo de saccharose est préféré aux autres aliments contenant, soit le glucose en quantité équivalente, soit les amylacés additionnés de saccharine (tab1. 6). Cette préférence se manifeste définitivement dès la première semaine après le sevrage (fig. I), car les animaux qui ont le choix consomment l'aliment contenant le sucre en quantité deux fois supérieure à celui contenant le glucose (appétibilité $=0,46$ ). Toutefois, on constate une certaine variabilité du comportement des porcelets, surtout dans le cas de l'aliment supplémenté en saccharine : en effet, les porcelets de l'une des quatre répétitions ont préféré l'aliment à base de saccharine alors que tous les autres ont, dès le départ, très nettement préféré l'aliment à base de saccharose. Lorsque les animaux ne reçoivent qu'un seul aliment (expérience 2), la variabilité des quantités consommées est considérablement réduite entre répétitions. Les quantités d'aliment consommées apparaissent remarquablement constantes, quel que soit le composé incorporé (saccharose ou glucose), et quel que soit le taux de ce dernier (tabl. 6). De plus, à quantité d'aliment constante consommée, correspond une vitesse de croissance identique dans tous les lots. L'indice de consommation est très voisin pour des animaux d'âge identique à ceux utilisés dans 1'expérience d'appétibilité; toutefois l'aliment contenant ro p. Ioo de glucose semble moins bien utilisé que les autres dans l'expérience 2 . 


\section{TABLEAU 5}

Influence respective du saccharose et du sirop de glucose à haute teneur en fructose (S.G.H.T.F.) sur la technologie de fabrication des a.iments de sevrage à 3 semaines (expérience 4) Influence of sucrose and H.F.C.S. on pelleting of the starter for 3 weeks weaning (experiment 4)

\begin{tabular}{|c|c|c|c|c|}
\hline $\begin{array}{l}\text { Aliment } \\
\text { lieed }\end{array}$ & \multicolumn{2}{|c|}{$\begin{array}{l}\text { Témoin } \\
\text { Control }\end{array}$} & \multicolumn{2}{|c|}{$\begin{array}{l}\text { Expérimental } \\
\text { Experiment storter }\end{array}$} \\
\hline Mode d'agglomération (Pelleting process) : & $\begin{array}{l}\text { sec }\left({ }^{1}\right) \\
\text { Dry } \\
\text { pelleted }\end{array}$ & 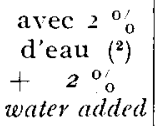 & $\begin{array}{l}\text { Sec } \\
\text { Dry } \\
\text { pelleted }\end{array}$ & $\begin{array}{l}\text { Sec } \\
\text { I)ry } \\
\text { polleted }\end{array}$ \\
\hline 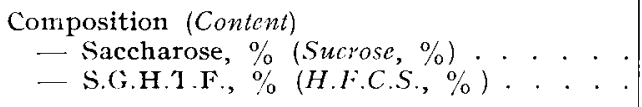 & $I_{4}$ & I4 & $\begin{array}{c}10,3 \\
5\end{array}$ & $\begin{array}{l}6,7 \\
10\end{array}$ \\
\hline 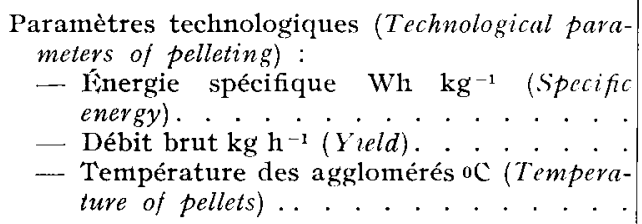 & $\begin{array}{l}29,7^{a} \\
-\left({ }^{1}\right) \\
5^{8 a}\end{array}$ & $\begin{array}{l}27,9^{b} \\
66^{a} \\
60^{a}\end{array}$ & $\begin{array}{l}27,2^{b} \\
77^{b} \\
54^{b}\end{array}$ & $\begin{array}{l}21,8^{c} \\
102^{r} \\
52^{b}\end{array}$ \\
\hline $\begin{array}{l}\text { Teneur en eau des agglomérés, \% (Moisture } \\
\text { content): } \\
\text { - Sortie filière (After pelleting) } \quad . \quad . \quad . \\
\text { - Après refroidissement (After cooling) . }\end{array}$ & $\begin{array}{l}7,1 \\
6,5\end{array}$ & $\begin{array}{l}8,4 \\
7,5\end{array}$ & $\begin{array}{l}8,4 \\
8,7\end{array}$ & $\begin{array}{l}10,4 \\
9,1\end{array}$ \\
\hline $\begin{array}{l}\left.\text { Dureté bar } \mathrm{cm}^{-1} \text { (Hardness bar } \mathrm{cm}^{-1}\right) \\
\text { Friabilité } \%(\text { Durability } \%) \\
\end{array}$ & $\begin{array}{l}3,6^{a} \\
4,8^{a}\end{array}$ & $\begin{array}{l}3,4^{n} \\
0,9^{b}\end{array}$ & $\begin{array}{l}3,2^{a} \\
0,7^{b}\end{array}$ & $\begin{array}{l}1,9^{b} \\
0,9^{b}\end{array}$ \\
\hline
\end{tabular}

(1) Donnée manquante : aliment difficile à agglomerer à sec (Missing zalue: fecd difficult to pellet as dry meal).

$\left({ }^{2}\right)$ Aliment utilisé comme témoin dans l'expérience 4 (Feed used as a control treatment in experiment 4).

$a, b, c$ : L $\mathbf{P}<0,0 \mathrm{I}$ (Means affected with the same subscript are not stastistically significant 1 . $0.0 \mathrm{I}$ ).

\section{4. - Appétibilité des aliments contenant le sirop de glucose (S.G.H.T.F.)}

Les aliments contenant le sirop de glucose riche en fructose (S.G.H.T.F.) sont toujours consommés en plus faible quantité que les aliments contenant le saccharose quel que soit l'âge des animaux et la durée d'allaitement. L'appétibilité est toujours inférieure à 0,7 par rapport à 1'aliment témoin et 1'on constate que 7 I p. Ioo des portées allaitées préfèrent l'aliment contenant le saccharose; 62 p. Ioo des mêmes portées sevrées continuent à préférer l'aliment sucré aux deux autres renfermant le sirop. L'appétibilité des aliments n'est pas modifiée lors du sevrage (tabl. 7 et figure 2). Mais, les quantités d'aliment consommées par animal varient 
TABLEAU 6

Appétibilité des aliments pour le porcelet sevré à 35 jours suivant la méthode expérimentale envisagée (expériences $I$ et 2 )

Infuence of glucose, sucrose or saccharin on the palatibility of starters by piglets weaned at 5 weeks according to the experimental method (experiments $I$ and 2 )

\begin{tabular}{|c|c|c|c|c|c|c|}
\hline $\begin{array}{c}\text { Conditions expérimentales } \\
\text { de } 5 \text { à } 9 \text { semaines } \\
\text { Experimental design } \\
5-9 \text { weeks }\end{array}$ & \multicolumn{3}{|c|}{$\begin{array}{c}\text { Expérience I } \\
\text { Rations au choix } \\
\text { (3 aliments) } \\
\text { Experiment I } \\
3 \text { diets as " free choice» }\end{array}$} & \multicolumn{3}{|c|}{$\begin{array}{l}\text { Expérience } 2 \\
\text { Rations imposées } \\
1 \text { aliment/groupe } \\
\text { Experiment } 2 \\
I \text { diet every group }\end{array}$} \\
\hline $\begin{array}{c}\text { Aliment à base de (Kind of } \\
\text { sweetner added) . . . . }\end{array}$ & $\begin{array}{l}\text { Saccha- } \\
\text { rose } 5 \% \\
\text { Sucrose }\end{array}$ & $\begin{array}{l}\text { Glucose } \\
5 \% \\
\text { Glucose }\end{array}$ & \begin{tabular}{|} 
Amylacés \\
$5 \%$ \\
$(0,22 \%$ de \\
saccharine \\
$(1)$ \\
Siarch \\
derivatives \\
+ saccha- \\
rin
\end{tabular} & $\begin{array}{l}\text { Saccha- } \\
\text { rose } 5 \% \\
\text { Sucrose }\end{array} \mid$ & $\begin{array}{l}\text { Glucose } \\
5 \% \\
\text { Glucose }\end{array}$ & $\begin{array}{l}\text { Glucose } \\
\text { Io \% } \\
\text { Glucose }\end{array}$ \\
\hline $\begin{array}{c}\text { Gain de poids, } g_{\text {in }} \mathrm{j} \text { (Daily } \\
\text { weight gain, g during the } \\
\text { test) } . \cdot \cdot \cdot \cdot \cdot \cdot \cdot\end{array}$ & & $34^{r}$ & & $\begin{array}{l}355 \\
(10)\end{array}$ & ${ }^{355}(\mathrm{r} 3)$ & $34^{\circ}$ \\
\hline $\begin{array}{l}\text { Quantité d'aliment consom- } \\
\text { mée, g/j (Average daily' feed } \\
\text { consumption) . . . . . . }\end{array}$ & $\begin{array}{l}4 \mathrm{I} 2^{a} \\
(44)\end{array}$ & $\begin{array}{l}71^{b} \\
\quad(47)\end{array}$ & $\begin{array}{l}\mathrm{I}_{4} \mathrm{I}^{b} \\
\quad\left(\mathrm{I}_{\mathrm{O}} \mathrm{O}_{3}\right)\end{array}$ & ${ }^{65} \mathrm{I}(7)$ & 644 (9) & ${ }^{655}(\mathrm{I} 8)$ \\
\hline $\begin{array}{l}\text { Indice de consommation. } \\
\text { (lood conversion ratio). }\end{array}$ & & I, 83 & & $\mathrm{I}, 8_{4}^{a b}(8)$ & $\begin{array}{r}\mathrm{I}, 82^{b} \\
(9)\end{array}$ & $\mathrm{I}, 94^{b}$ \\
\hline
\end{tabular}

(1) I Io $\mathrm{mg} / \mathrm{kg}$ d'aliment granulé à $2,5 \mathrm{~mm}$ ( I I O $\mathrm{mg} / \mathrm{kg}$ of pelleted feed $2.5 \mathrm{~mm} \varnothing)$.

() Coefficient de variation du parametre, $\mathrm{p}$. Ioo (Coefficient of variation of the mean, $p$. Ioo).

très fortement : ainsi le coefficient de variation interportées pour les 3 aliments testés varie suivant la semaine entre 96 et I30 p. Ioo avant sevrage quel que soit l'âge des animaux, et de 47 à 86 p. Ioo seulement après sevrage. Cependant, les valeurs trouvées après le sevrage (fig. 2) semblent montrer que la hiérarchie établie au cours de la période d'allaitement n'est pas remise en question et que la préférence pour l'aliment témoin à base de saccharose est définitive.

On peut noter que la consommation relative des aliments expérimentaux contenant des proportions croissantes de sirop varie faiblement et que l'animal de 3 ou de 4 semaines porte sa préférence sur l'aliment qui ne contient que du saccharose même à faible taux (fig. 2). 


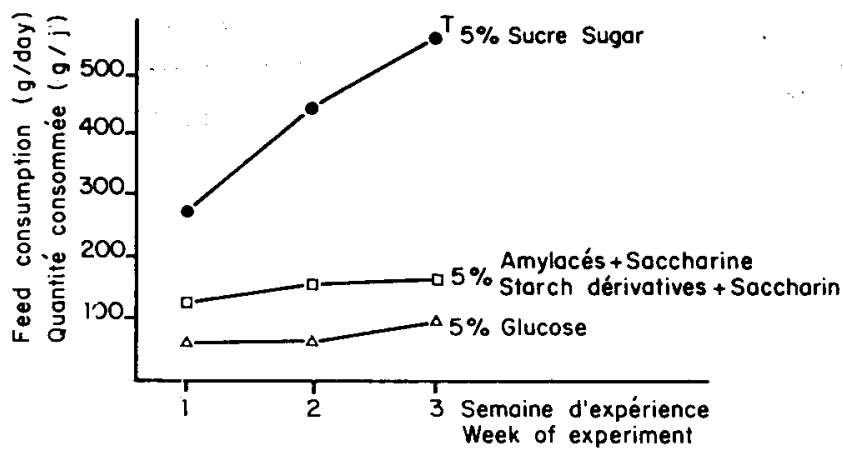

FIG. I. - Évolution de la préférence alimentaire des porcelets eflectuant un choix entre 3 aliments. Evolution of the palatability of starters for animals receiving 3 test feed.

\section{TABLEAU 7}

Évolution de l'appétibulité (1) des aliments contenant du sirop de glucose à haute teneur en fructose suivant l'âge et le type de sevrage (expériences 3 et 4 )

Evolution of the palatability ratio of the feed containing H.F.C.S. according to age and weaning time (experiments 3 and 4)

\begin{tabular}{|c|c|c|c|c|c|c|}
\hline $\begin{array}{l}\text { Age au } \\
\text { sevrage } \\
\text { Age at } \\
\text { weaning }\end{array}$ & $\begin{array}{l}\text { Période (Period) } \\
\text { Aliment à base de : } \\
(\text { Feed based on })\end{array}$ & $\left|\begin{array}{c}2-3 \\
\text { semaines } \\
2-3 \quad \text { weeks }\end{array}\right|$ & $\begin{array}{c}3-4 \\
\text { semaines } \\
\text { 3-4 weeks }\end{array}$ & $\begin{array}{c}4-5 \\
\text { semaines } \\
4-5 \text { weeks }\end{array} \mid$ & $\begin{array}{c}5-6 \\
\text { semaines } \\
5-6 \text { weeks }\end{array}$ & $\begin{array}{l}\text { Appétibilité } \\
\text { (période } \\
\text { totale) } \\
\text { Palatability } \\
\text { ratio } \\
\text { (total period) }\end{array}$ \\
\hline $\begin{array}{c}5 \text { semaines } \\
5 \text { weeks }\end{array}$ & \begin{tabular}{cc} 
& \multicolumn{2}{c}{ P. Ioo } \\
Saccharose & S.G.H.T.F. \\
Sucrose & H.F.C.S. \\
5 & - \\
- & 7,3 \\
- & $\mathrm{1} 2$
\end{tabular} & $\begin{array}{l}\mathrm{x} \\
0,60 \\
0,40\end{array}$ & $\begin{array}{l}\text { I } \\
0,7 \mathrm{I} \\
0,34\end{array}$ & $\begin{array}{cc}\mathrm{I} & \\
0,56 & \text { Sevr } \\
0,5^{2} & \text { Weal }\end{array}$ & $\begin{array}{ll} & \mathbf{I} \\
\text { rage } & 0,68 \\
\text { ining } & 0,69\end{array}$ & $\begin{array}{l}\mathbf{I} \\
0,69 \\
0,64\end{array}$ \\
\hline $\begin{array}{c}3 \text { semaines } \\
3 \text { weeks }\end{array}$ & \begin{tabular}{cc}
\multicolumn{2}{c}{ p. 100} \\
Saccharose & S.G.H.T.F. \\
Sucrose & H.F.C.S. \\
I4 & - \\
I0,5 & 5 \\
7 & I0
\end{tabular} & $\begin{array}{cc}\text { I } & \\
0,54 & \text { Sev } \\
0,89 & W e a\end{array}$ & $\begin{array}{ll}\qquad \text { I } \\
\text { rage } & 0,78 \\
\text { aning } & 0,67\end{array}$ & $\begin{array}{l}\mathrm{I} \\
0,4 \mathrm{I} \\
0,40\end{array}$ & - & $\begin{array}{l}\text { I } \\
0,48 \\
0,47\end{array}$ \\
\hline
\end{tabular}

(1) Appétibilité : $\frac{\text { Quantité d'aliment expérimental consommée. }}{\text { Quantité d'aliment témoin }}$

Palatability : $\frac{\text { Total amount of experimental feed consumption. }}{\text { Total amount of control feed }}$ 


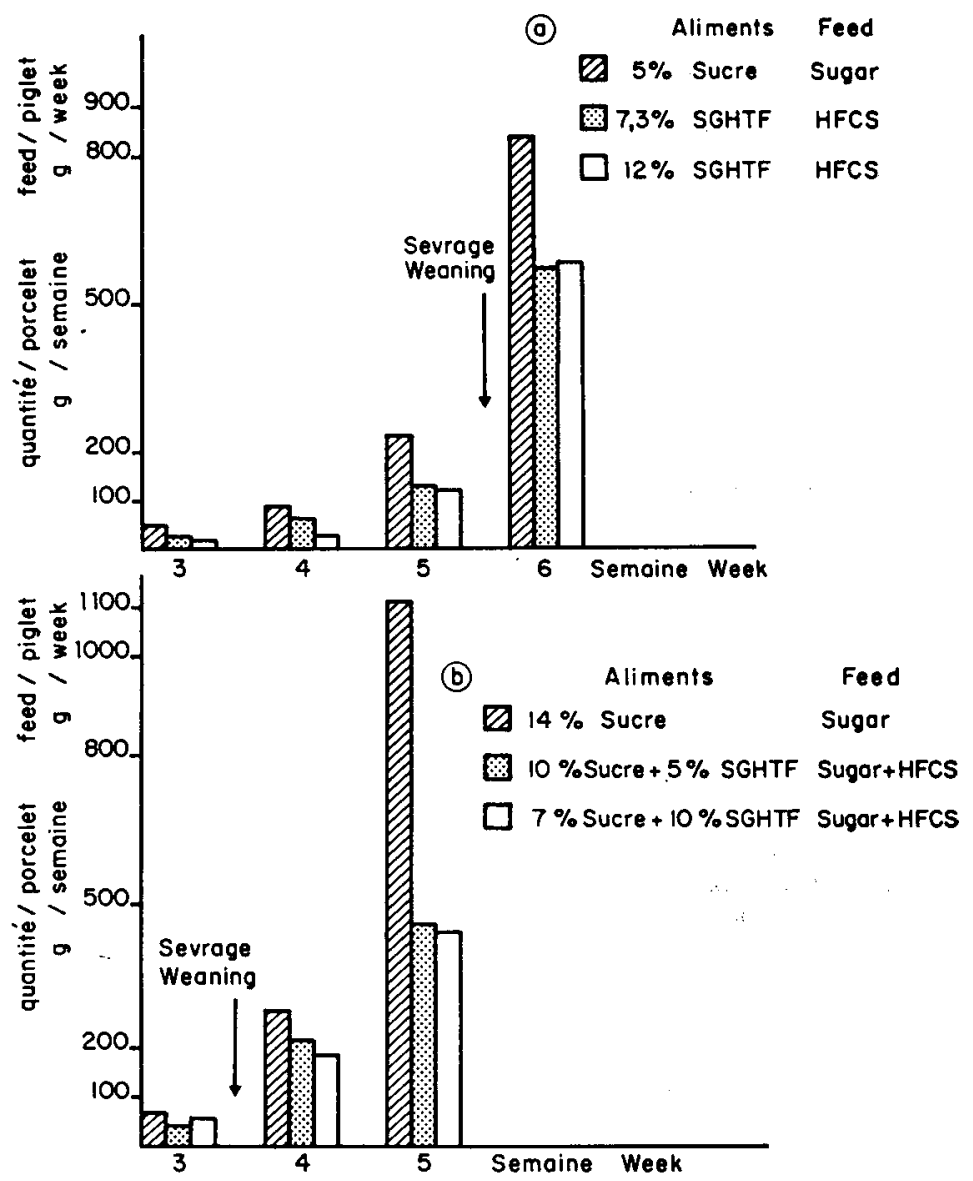

FIG. 2. - Quantités d'aliments consommées par semaine et par porcelet suivant l'âge et le taux de sirop de glucose.

Weekly food consumption per piglet according to age and level of H.F.C.S.

a) Porcelets sevrés à 5 semaines.

b) Porcelets sevrés à 3 semaines.

a) Piglets weaned at 5 weeks.

b) Piglets weaned at 3 weeks.

\section{IV. - Discussion et conclusion}

Les dosages colorimétriques du glucose vrai (glucose oxydase) et du fructose sont suffisants pour caractériser le S.G.H.T.F. et contrôler sa qualité. Mais, l'identification quantitative des glucides simples (glucose, fructose, saccharose) dans le mélange d'aliment s'avère impossible à l'aide des seules méthodes colorimétriques. Le dosage des sucres réduisant le ferricyanure dans l'aliment témoin peut rendre compte de plusieurs composés : présence de glucose dans les matières premières, libération possible de glucose et de fructose provenant de 1'hydrolyse partielle de saccharose pendant l'agglomération (MeLcion et al., r974), apport de lactose 
par le lait écrémé, présence de substances réductrices non glucidiques. Ainsi, il n'est pas possible de caractériser l'introduction de S.G.H.T.F. dans les aliments par un dosage colorimétrique. Une caractérisation quantitative à l'aide des techniques de chromatographie serait nécessaire afin de déceler la présence de sirop dans une farine complète pour porcelets.

L'incorporation de saccharose dans les aliments pour le porcelet a été surtout recommandée (Nerson, Hazei, et CATRON, I953; I,EWIS, Catron et CombS, I953) au moment où les disponibilités de ce produit sur le marché mondial étaient grandes. Par ailleurs, la préférence du porcelet pour les aliments secs contenant du saccharose et le refus d'aliments de composition identique n'en contenant pas, dans le cas où les animaux peuvent effectuer un choix entre deux aliments, avaient pu faire croire à la nécessité d'en incorporer au moins $5 \mathrm{p}$. Ioo dans les premiers aliments de démarrage (LEwis, Catron et Combs, I953; SALMON-LEGaGneur et FÉvriér, r956). Mais, le fait que le porcelet est susceptible de préférer, parmi les aliments " sucrés ", un aliment renfermant jusqu'à 40 p. roo de saccharose (ComBs et WALIACE, I959), démontre le danger de l'extrapolation des notions qualitatives d'appétibilité à la pratique de l'alimentation des jeunes animaux (WAHLSTROM, HAUSER et IrbaL, I974; AUmaITRE, I975).

Ainsi les animaux, qui penvent porter leur choix dans le seul cas expérimental sur un aliment renfermant du saccharose, délaissent les aliments à base de glucose incorporé à 5 ou à ro p. Ioo, ainsi que les aliments supplémentés en saccharine, comme l'ont montré Notzor, et al., I955. Ils semblent avoir un comportement tout différent lorsqu'ils n'ont qu'un seul aliment; dans ces dernières conditions toujours rencontrées dans la pratique de l'élevage, la quantité d'aliment consommée est très exactement la même que celle observée pour l'aliment "sucré ".

Par ailleurs, nous observons que les aliments renfermant le sirop de glucose (S.G.H.T.F.) ne sont pas aussi appétibles que ceux renfermant une quantité équivalente de saccharose. De même, les animaux répondent toujours défavorablement par leur choix à une diminution de la teneur en saccharose (de I4 à 7 p. roo) dans l'aliment, même si 1'on compense la différence par du S.G.H.T.F. 'Toutefois, les animaux ne semblent pas réagir en fonction des caractéristiques technologiques de l'aliment telles que la dureté ou la friabilité. Ainsi, pour des agglomérés dont la dureté varie significativement entre 3,2 et $\mathrm{I}, 9$ bar $/ \mathrm{cm}^{-1}$, les quantités d'aliment consommées sont identiques (fig. 2). Dans tous les cas, la proportion d'aliments à base de saccharose dépasse $60 \mathrm{p}$. Ioo de la consommation totale de 1'aliment considérée comme seuil minimum significatif de la préférence par BAI,DWIN (I976). L'incorporation de fortes quantités de saccharose pose toujours des problèmies technologiques à l'agglomération : l'énergie spécifique consommée est élevée mème après addition d'eau et le débit de la presse considérablement réduit. Le sirop de glucose (S.G.H.T.F.) améliore incontestablement les conditions de fabrication, mais, incorporé à plus de 5 p. Ioo, outre qu'il apporte une forte quantité d'eau, il diminue la dureté et la tenue des agglomérés.

En conclusion, nos résultats montrent que le porcelet préfère le saccharose au glucose et à ses dérivés (S.G.H.'T.F.) lorsqu'on en incorpore dans la ration. Mais la nécessité d'incorporer des produits à pouvoir sucrant aux aliments du porcelet sous la mère, et aux aliments de sevrage précoce peut non seulement être discutable au plan économique, mais encore au plan de la stimulation éventuelle du montant et de la précocité de la prise alimentaire, qui n'est presque jamais démontrée. Par ailleurs, le comportement du porcelet vis-à-vis des produits sucrés nous paraît particulier. Ainsi, les animaux qui semblent répondre favorablement 
à la consommation d'une quantité équivalente d'eau sucrée ou d'eau glucosée (KENNEDY et BALDWTN, I972), délaissent l'aliment sec renfermant du glucose au profit de celui contenant le saccharose.

Par ailleurs, on peut dire que le porcelet s'écarte d'un aliment dans lequel on a incorporé du sirop de glucose à haute teneur en fructose au profit de l'aliment à base de saccharose, par une réaction buccopharyngée au goût de l'aliment plutôt que par une régulation d'origine métabolique. Enfin, on peut se demander si au cours de l'isomérisation industrielle du glucose, d'autres composés ne sont pas formés puisque $4 \mathrm{p}$. Ioo au moins de la matière sèche des produits testés ne peuvent être assimilés à la fraction "sucres réducteurs " (tabl. 4), modifiant le goût du produit final. Dans notre essc $i$, le porcelet ne réagit pas favorablement à la présence de S.G.H.T.F. ou au goût qu'il confère à l'aliment. Ce produit ne peut donc être substitué au saccharose, que l'on continue à incorporer plus par habitude que par nécessité aux aliments de sevrage précoce.

Accepté pour publication en mai 1978 .

\section{Remerciements}

A la Société des Produits du Mais, MM. Dumond et ThIEUd, ín, 92 i 40 Clamart;

A l'Union des Coopératives d'Alimentation du Bétail, MM. COLLET et LERICOLLAIS, O240o Château-Thierry, pour leur participation à l'expérience.

\section{Summary}

Use of glucose, fructose rich glucose syrup (H.F.C.S.) or sucrose in diets of early weaned piglets: infuence on pelleting and palatability

The effect of incorporating sweet substances (sucrose, saccharin, fructose rich glucose syrup or (H.F.C.S.) into piglet feeds upon the palatability of the latter was measured during 4 experiments using 252 animals weaned at 35 days and 80 animals weaned at $2 \mathrm{I}$ days.

The incorporation of sucrose reduced the pelleting yield; use of $5 \mathrm{p}$. I00 H.F.C.S. increased the rate of passage of the feeds at pelleting, but with io $\mathrm{p}$. Ioo, the hardness of the pellets decreased from 3.6 bar $\mathrm{cm}^{-1}$ to 1.9 bar $\mathrm{cm}^{-1}$.

When the animals had the choice between 3 diets containing $5 \mathrm{p}$. Ioo sucrose, $5 \mathrm{p}$. I oo glucose or 5 p. roo starchy substances + saccharin, their daily mean intake was 4 I $2,7 I$ and I 4 I g, respectively. These results clearly show that the animals prefer sucrose containing feeds. When increasing levels of H.X.C.S. were incorporated into the diets, the animals still preferred those containing the sucrose. Thus, H.F.C.S. is not as palatable as sucrose although their molecular composition is similar. The palatability ratio only represented 0.69 for the piglets weaned at 5 weeks versus 0.48 for those weaned at 3 weeks.

However, when only one diet was available for each group of piglets, no difference was observed between glucose and sucrose; the average feed intake was 644 or $65 \mathrm{I} \mathrm{g} / \mathrm{day} / \mathrm{piglet}$ during the experimental period.

Accordingly, the notion of palatability is an experimental artifice and values recorded when two diets were offered do not allow to conclude in favour of an incorporation of sucrose or sweet substances into the diets of early weaned piglets. 


\section{Références bibliographiques}

Aumaitre A., I975. Sur l'appétibilité des aliments destinés aux porcelets : importance du sel et du sucre. Journées Rech. Porcine en France, I.N.R.A.-I.T.P. éd., Paris, 7, i69-1 76.

Bald win B. A., 1976. Quantitative studies on taste preference in pigs. Proc. Nutr. Soc., 35, 69-73.

Combs G. E. Jr., Watidice H. D., i969. Palatable creep feeds for pigs. Fla. Agri. Expt. Sta. Bull., 610, I-I 2 .

Delor't-Laval J., DRevet S., I97o. Méthodes d'appréciation de la dureté des fourrages agglomérés. Ind. Alim. Anim., 23, 43-54.

Iisuka H., Ayukawa Y., SuEkane M., Kanno M., 1972. Verfahren zur Herstellung einer Enzymzubereitung und ihre Verwendung zum Isomerisieren von Glucose. Hauptpatent. Patenschrift $n^{0} 5 I 5992$. C.P.C. Inter. Inc. Englewood Cliffs, N. J. U.S.A., I-I 4.

KENNEDY J. M., BALDWIN B. A., I972. Taste preferences in pigs for nutritive and non nutritive sweet solutions. Anim. Behaviour, 20,706-7 8 .

LE MAGNEN J., I962. Vocabulaire technique des caractères organoleptiques et de dégustation des produits alimentaires. Ann. Nutr. Alim. Paris, 11, B $\mathbf{1}_{\mathbf{1}} \mathbf{B}_{\mathbf{8 6}}$.

Lewis C. J., Catron D. V., Combs G. E., I953. Sugar in pigs starter. J. Anim. Sci., 12, 19231938 .

Mercion J. P., Vaissade P., Valdebovze P., Viroben G., I974. Influence des conditions d'agglomération sur quelques caractéristiques physico-chimiques d'un aliment pour porcelets. Ann. Zootech., 23, 149-160.

Nelson L. F., HazEI. L. N., Catron D. V., I953. Baby pigs have a sweet tooth. Feedstuffs, 25 (I2), I2-I 8.

Notzold R. A., BeCKER D. F., Terril, S. W., JENSEN A. H., i955. Saccharin and dried cane molasses in swine rations. J. Anim. Sci, 14, 1068-1072.

PFost H. B., AII,EN R. N., I 962 . A standard method of measuritng pellet durability. Proc. peed prod. school, Kansas City, I2-I4 nov, 25-29.

SALMON-LEGAGNEUR E., FEVRIER R., I956. Les préférences alimentaires du porcelet. II. Le sucre dans les aliments pour porcelets. Ann. Zootech., 5, 87-97.

SchUlTZ R., I965. Uber das Messen der mechanischen Festigkeit von gepressten Mischfutter. Die Mïhle, 102, I47-155.

VAissade P., Aumaitre A., Rerat A., I973. Mesure en continu de la glycémie du sang porte et périphérique chez le porc éveillé : application à l'étude de l'absorption intestinale des glucides. Symposium Inter. Pharm. Technicon., 4-5 oct. I973, Paris, 18 pp.

WAHLSTrom R. C., HAUSER I. A., LiBaL G. W., i974. Effect of low lactose whey, skim-milk and sugar on diet palatability and performance of early weaned pigs. $J$. Anim. Sci., 38, I 267I 27 I. 